\title{
NEW CONSTANTS FOR SAMPSON-LIESKE THEORY OF THE GALILEAN SATELLITES OF JUPITER FROM MUTUAL OCCULTATION DATA
}

\author{
R. VASUNDHARA \\ Indian Institute of Astrophysics, Bangalore 560034, India \\ AND \\ J.E. ARLOT AND P. DESCAMPS \\ Bureau des Longitudes, URA 0707 CNRS, \\ 77 avenue Denfert-Rochereau, 75014 Paris, France
}

\begin{abstract}
New constants for Sampson-Lieske theory of the Galilean Satellites have been derived using 6360 individual photographic positions (18911990 ) and 438 pseudo astrometric positions from mutual occultations during $1973,1979,1985$ and 1991 . Using these new sets of constants, significant improvement is noticed in the $\mathrm{O}-\mathrm{C}$ values of the sky plane coordinates of the mutual event data set and residuals in longitude for Io and Europa are found to improve. Problems concerning the inclusion of mutual event data in attempting evaluation of secular variations of the mean motions of the satellites are discussed.
\end{abstract}

\section{Introduction}

The constants of motion of the Galilean Satellites are efficiently updated using Lieske's $(1974,1977)$ technique by computing the corrections $(\varepsilon, \beta)$ to the basic sets of constants. Lieske progressively updated these constants from E1 (Lieske, 1978), using the eclipse data of 1878-1903, to E2 (Lieske, 1980), by supplementing visual eclipses between 1903 and 1972, photographic data between 1967 and 1978 and mutual events of 1973 . The E2x3 (Lieske, 1987) ephemeris was derived by further additions of more mutual event pairs from 1973 and 1979, 183 pairs of data from Voyager optical navigation images and 15711 classical eclipses from 1652-1983. Arlot (1982) 
used 8856 individual photographic observations between 1891 to 1978 to derive the G5 ephemeris. The present investigations relate to determinations of new sets of constants using photographic data between 1891 to 1990 and astrometric positions derived from mutual occultations during 1973, 1979, 1985 and 1991.

\section{The observational material}

Most of the photographic data that were used by Arlot(1982) were utilized in this study. Additional excellent unpublished photographic material covering the period between 1986 and 1990 (Pascu, private communication) have been included. Table 1 gives the details of the observations and includes the mutual occultation data set containing the astrometric positions derived from the mutual occultation light curves of 1985 and 1991 assuming Hapke's light scattering law for a macroscopically rough surface (Hapke, 1984), using the Hapke's parameters derived by Descamps and Thuillot (1993). The published positions of the 1985 series by the Galilean Satellite Observers (GSO and Franklin, 1991), which were derived using Lambert's law, were reconstructed to adopt Hapke's law and included in our studies (Vasundhara, 1993 and 1994). Published positions of 1973 (Aksnes and Franklin 1976) and 1979 (Aksnes et al. 1984) were also used after accounting for the phase corrections (Aksnes, Franklin and Magnusson 1986). Table 1 gives the statistics for all the data that we used.

\section{Corrections to the constants}

The method developed by Arlot(1982) has been used in the present study. The starting ephemeris was E1 (Lieske 1987) and 3 more iterations were carried out by progressively adjusting the $(\epsilon, \beta)$ values. The constants that were updated were the mean motions $\left(\epsilon_{6}-\epsilon_{8}\right)$, the primary eccentricities $\left(\epsilon_{16}-\epsilon_{19}\right)$, the primary sine inclinations $\left(\epsilon_{21}-\epsilon_{24}\right)$, the mean longitudes $\left(\beta_{1}, \beta_{2}, \beta_{4}\right)$, the proper perijoves $\left(\beta_{6}-\beta_{9}\right)$, the proper nodes $\left(\beta_{11}-\beta_{14}\right)$, the libration amplitude $\left(\epsilon_{9}\right)$ and the libration phase angle $\left(\beta_{5}\right)$.

Let us remind that the G5 ephemeris was based only on photographic observations from 1891 to 1978 . By adding the recent photographic data made from 1986 to 1990 (Pascu, private communication) and the mutual occultations data (see table 1) with a weight of 50 , we obtained the ephemeris I32, and I33 using a weight of 10 . The ephemeris G6 was constructed using only the photographic data of Table 1 and the updated $\varepsilon$ and $\beta$ values for Jupiter related constants and mass of the satellites introduced by Lieske (1987) in his E2x3 ephemerides. The values of the constants for all these ephemerides are available using FTP anonymous on 
TABLE 1. Details on the data

\begin{tabular}{|c|c|c|c|c|c|c|c|c|c|c|}
\hline \multirow[t]{2}{*}{ Observers } & \multirow[t]{2}{*}{ Codes $^{1}$} & \multirow[t]{2}{*}{ Year } & \multicolumn{4}{|c|}{ No. of positions } & \multicolumn{4}{|c|}{ r.m.s. of the residuals (arcsec } \\
\hline & & & $\mathrm{J} 1$ & $\mathrm{~J} 2$ & J3 & $\mathrm{J} 4$ & G5 & G6 & I32 & I33 \\
\hline Renz & $\mathrm{H}, \mathrm{P}$ & $1891-1898$ & 171 & 175 & 184 & 174 & 0.117 & 0.111 & 0.110 & 0.111 \\
\hline Balanovsky & $\mathrm{P}$ & 1904-1910 & & & & & & & & \\
\hline Chevalier & Zo-Se & $1917 / 1918$ & 132 & 132 & 133 & 121 & 0.148 & 0.150 & 0.151 & 0.151 \\
\hline De Sitter & G & $1918 / 1919$ & & & & & & & & \\
\hline De Sitter & $\mathrm{C}$ & 1924 & & & & & & & & \\
\hline Petrescu & B & 1934 & 85 & 104 & 106 & 118 & 0.217 & 0.222 & 0.223 & 0.222 \\
\hline Petrescu & $\mathrm{P}$ & 1936 & & & & & & & & \\
\hline Van Biesbr. & $\mathrm{Y}$ & $1961-1963$ & & & & & & & & \\
\hline Gorel & $\mathrm{N}$ & $1962-1966$ & & & & & & & & \\
\hline Soulié & Bo & $1966-1974$ & 284 & 282 & 318 & 307 & 0.390 & 0.390 & 0.390 & 0.390 \\
\hline Gorel & $\mathrm{N}$ & $1973-1974$ & & & & & & & & \\
\hline Debehogne & RJ,U,LS & $1977 / 1978$ & & & & & & & & \\
\hline Ianna et al. & $\mathrm{Mc}-\mathrm{C}$ & $1977 / 1978$ & 70 & 100 & 95 & 108 & 0.104 & 0.104 & 0.104 & 0.104 \\
\hline Pascu & $\mathrm{Mc}-\mathrm{C}$ & $1967 / 1968$ & 88 & 87 & 89 & 95 & 0.095 & 0.095 & 0.095 & 0.095 \\
\hline$"$ & USNO & 1973 & 72 & 65 & 62 & 67 & 0.111 & 0.110 & 0.111 & 0.111 \\
\hline$"$ & $"$ & 1974 & 107 & 115 & 120 & 123 & 0.082 & 0.085 & 0.085 & 0.085 \\
\hline$"$ & $"$ & $1975-1977$ & 107 & 116 & 109 & 119 & 0.085 & 0.086 & 0.086 & 0.086 \\
\hline$"$ & $"$ & $1977 / 1978$ & 59 & 59 & 53 & 59 & 0.102 & 0.103 & 0.103 & 0.103 \\
\hline $\mathrm{Pascu}^{2}$ & $"$ & 1986 & 84 & 74 & 82 & 80 & 0.077 & 0.074 & 0.074 & 0.074 \\
\hline$"$ & $"$ & 1987 & 116 & 133 & 129 & 133 & 0.074 & 0.070 & 0.071 & 0.071 \\
\hline$"$ & $"$ & $1988 / 1989$ & 52 & 54 & 60 & 60 & 0.059 & 0.056 & 0.056 & 0.056 \\
\hline$”$ & $"$ & 1990 & 79 & 72 & 84 & 96 & 0.068 & 0.066 & 0.067 & 0.067 \\
\hline $\mathrm{AkFr}^{3}$ & - & 1973 & 45 & 48 & 3 & 0 & 0.017 & 0.014 & 0.014 & 0.014 \\
\hline $\mathrm{Ak}^{4}$ & - & 1979 & 4 & 7 & 3 & 0 & 0.010 & 0.014 & 0.011 & 0.011 \\
\hline $\mathrm{Ar}^{5}, \mathrm{GSOF}^{6}$ & - & 1985 & 59 & 62 & 73 & 26 & 0.024 & 0.024 & 0.024 & 0.024 \\
\hline $\mathrm{Ar}^{7}$ & - & 1991 & 45 & 53 & 9 & 1 & 0.036 & 0.024 & 0.028 & 0.030 \\
\hline
\end{tabular}

1. Observatory codes. H:Helsingfors, P:Pulkovo,G:Greenwich C:The Cape, P:Paris, Y:Yerkes, N:Nicolaiev, Bo:Bordeaux, RJ:Rio de Janeiro, U:Uccle, B:Bucarest, LS:La Silla, Mc-C:Mc Cormick, USNO:U.S. Naval Observatory, Washington D.C.

2. Private communication

3. Aksnes and Franklin, 1976

4. Aksnes et al., 1984

5. Arlot et al., 1992

6. GSO and Franklin, 1991

7. Arlot et al., 1996 
TABLE 2. Error in Longitude (minutes of time)

\begin{tabular}{lccccc}
\hline Data set & J1 & J2 & J3 & J4 & Ephem. \\
\hline Ph & $0.060 \pm 0.011$ & $0.009 \pm 0.012$ & $0.053 \pm 0.015$ & $0.073 \pm 0.020$ & G5 \\
Ph+ME & $0.058 \pm 0.010$ & $-0.021 \pm 0.011$ & $0.041 \pm 0.014$ & $0.076 \pm 0.019$ & G5 \\
\hline Ph & $0.019 \pm 0.011$ & $0.038 \pm 0.012$ & $0.058 \pm 0.015$ & $0.207 \pm 0.020$ & G6 \\
Ph+ME & $0.019 \pm 0.010$ & $0.038 \pm 0.012$ & $0.058 \pm 0.015$ & $0.207 \pm 0.019$ & G6 \\
\hline Ph & $0.019 \pm 0.011$ & $0.039 \pm 0.012$ & $0.078 \pm 0.015$ & $0.201 \pm 0.020$ & I32 \\
Ph+ME & $0.018 \pm 0.010$ & $0.017 \pm 0.011$ & $0.067 \pm 0.014$ & $0.203 \pm 0.019$ & I32 \\
\hline $\mathrm{Ph}$ & $0.021 \pm 0.011$ & $0.040 \pm 0.012$ & $0.059 \pm 0.015$ & $0.204 \pm 0.020$ & I33 \\
$\mathrm{Ph}+\mathrm{ME}$ & $0.021 \pm 0.010$ & $0.039 \pm 0.012$ & $0.059 \pm 0.014$ & $0.205 \pm 0.019$ & I33 \\
\hline $\mathrm{Ph}+\mathrm{ME}$ & 1508 & 1568 & 1624 & 1660 & data points \\
$\mathrm{Ph}+\mathrm{ME}$ & $1508+153$ & $1568+170$ & $1624+88$ & $1660+27$ & data points \\
\hline
\end{tabular}

TABLE 3. Secular accelerations of the Galilean satellites $\mathrm{J} 1, \mathrm{~J} 2, \mathrm{~J} 3$ in $\dot{n} / n \times 10^{11} \mathrm{yr}^{-1}$ units

\begin{tabular}{lccc}
\hline Authors & \multicolumn{1}{c}{$\mathrm{J} 1$} & $\mathrm{~J} 2$ & $\mathrm{~J} 3$ \\
\hline De Sitter (1928) & 25 & 27 & -16 \\
Brouwer \& Clemence (1961) & 32 & 27 & 16 \\
Greenberg (1986) & $32 \pm 8$ & $-16 \pm 4.5$ & $-16 \pm 4.5$ \\
Lieske (1987) & $-0.74 \pm 0.87$ & $-0.82 \pm 0.97$ & $-0.98 \pm 1.53$ \\
Goldstein (1996a) & $45.4 \pm 9.5$ & - & - \\
Goldstein (1996b) & $70 \pm 75$ & $56 \pm 57$ & $28 \pm 20$ \\
This paper/G5 & $24.6 \pm 7.3$ & $-12.7 \pm 8.4$ & $-0.22 \pm 10.7$ \\
This paper/I32 & $22.7 \pm 7.9$ & $-6.1 \pm 9.3$ & $+10.6 \pm 10.6$ \\
\hline
\end{tabular}

ftp.bdl.fr/pub/ephem/satel/theories or may be sent upon request near the authors.

\section{Results and discussion}

The r.m.s. of the residuals (table 1) shows a small improvement after using the mutual occultations data to fit the theory. More, it is remarkable that G6, which is fitted only on photographic data, shows very small residuals for the mutual occultations data. In any case, these data are of high accuracy. In table 2, we tried to determine a longitude shift from the residuals 
after different fits of the theory. This shift may be interpreted either as an error on the longitude at the origin of time, or as an error on the mean motion. It is puzzling to see that this error is decreasing when including recent observations to fit the theory (G6, I32, I33) for J1, stable for J3 but increasing slightly for $\mathrm{J} 2$ and much more for $\mathrm{J} 4$. On the other hand, the comparison between G6 and I32 shows that the mutual occultations data do not improve the ephemeris more than the photographic data for the period 1986-1993. This could be explained by the larger number of photographic data (1388 points) than the one of mutual occultations data (328 points). Table 3 gives the secular accelerations calculated from the residuals of all the data presented in table 1 calculated using G5 and I32. Our results are significant only for $\mathrm{J} 1$. Note that the methods of calculations of the other authors are completely different and that we give their data only to appreciate the scale of values of these accelerations.

In conclusion, the residuals of the mutual occultations data look very good but these data are not yet sufficient to improve the ephemerides. We look forward to using data from mutual eclipses and from the forthcoming mutual event opportunity in 1997.

\section{Acknowledgements}

We would like to thank Dr.J.H.Lieske from JPL for the computer codes for calculating the revised Sampson theory. We are indebted to Dr.D. Pascu from USNO for allowing us to use his yet unpublished excellent data of 1986-1990.

\section{References}

Aksnes, K., Franklin, F. 1976, Astron. J., 81, 464.

Aksnes, K., Franklin, F., Millis, R., Birch, P., Blanco, C., Catalano, S., Piironen, J. 1984, Astron. J., 89, 280.

Aksnes, K., Franklin, F., Magnusson, P. 1986, Astron. J., 92, 1436.

Arlot, J.E. 1982, Astron. Astrophys., 107, 305.

Arlot, J.E. et al., 1992, Astron. Astrophys. Supp. Series, 92, 151.

Arlot, J.E. et al., 1996, to be published in Astron. Astrophys. Supp. Series.

Brouwer, D., Clemence, G.M. 1961, in Planets and Satellites, Kuiper and Middlehurst ed., Chicago University Piers, p. 88.

Descamps, P., Thuillot, W. 1993, Icarus, in press.

de Sitter, W., 1928, Leiden Annals, 16, 92.

GSO and Franklin, F., 1991, Astrophys. 102, 806.

Goldstein, S.J., Jacobs, K.C. 1996, On the Evolution of the galilean satellites, in press.

Goldstein, S.J., Jacobs, K.C. 1996, A recalculation of the secular acceleration of IO, in press.

Greenberg, R., Goldstein, S.J., Jacobs, K.C. 1986, Nature, 323, 789.

Hapke, B.W. 1984, Icarus 59, 41.

Lieske, J.H., 1974, Astron. Astrophys. 31, 137.

Lieske, J.H., 1977, Astron. Astrophys. 56, 333. 
Lieske, J.H., 1978, Astron. Astrophys. 65, 83.

Lieske, J.H., 1980, Astron. Astrophys. 82, 340.

Lieske, J.H., 1987, Astron. Astrophys. 176, 146.

Vasundhara, R. 1993, Ph. D. Thesis.

Vasundhara, R. 1994, Astron. Astrophys. 281, 565. 